\title{
A Digital Implementation of Delay Relevant Frequency Measurement Technology Based on CORDIC Algorithm Xue Chen Qin ${ }^{1, a^{*}}$, Zhen Feng Pan ${ }^{2, b}$ and Jia Qi Zhang ${ }^{3, c}$ \\ ${ }^{1}$ Hohai University, Nan Jing, China \\ ${ }^{2}$ Hohai University, Nan Jing, China \\ ${ }^{3}$ Hohai University, Nan Jing, China \\ a vincent_qin1210@163.com, b tiancileo@126.com,,zhang_jiaqi01@163.com
}

\section{Keywords: EW ; DIFM ; CORDIC ALGORITHM ; MATLAB; FPGA}

\begin{abstract}
As one of the key technologies of radar electronic warfare systems, Digital Instantaneous Frequency Measurement (DIFM) technology need to measure signals intercepted quickly. The traditional method of instantaneous frequency measurement is to translate the frequency information into phase information to complete the measurement of the frequency. This paper presents a modified phase measurement method which uses CORDIC algorithm, which is suitable for implementation in FPGA for avoiding using the multipliers. On this basis, the frequency of the signal can be calculated, using MATLAB simulation tool to test its performance, and finally, this method is implemented in the FPGA simulation. The simulation results show that this method has high accuracy, good benefits instantaneous frequency measurement for single-frequency signal, suitable for modern electronic warfare digital receiver digital instantaneous frequency measurement needs.
\end{abstract}

\section{Introduction}

In a complex electromagnetic environment of modern electronic countermeasures, it is required that a microwave receiver is able to measure the frequency of an intercepted microwave signal over a large bandwidth, which has been the focus of worldwide research technology [1]. Due to the carrier frequency is a very important parameter, so in most of the electronic warfare system, the instantaneous frequency measurement receiver is often used for the reconnaissance of radar carrier frequency [2]. For single carrier pulse radar system, the receiver is required to measure the frequency of the carrier in a timely manner [3]. If the result is not located in the frequency of the system requirements, then the calibration parameters need to be provided immediately [4].

In this letter, we propose and demonstrate a new method to realizing DIFM based on CORDIC operations. This method is suitable for the measurement of single frequency signals with high precision and fast frequency. At the same time, this method can also be applied to the frequency modulation parameters of linear frequency modulation signal measurement.

\section{Principle}

Delay Line Correlator Instantaneous Frequency Measurement. The main idea of Instantaneous frequency measurement technology is to convert frequency information to phase information in order to we can measure it directly [5]. In the traditional methods, delay line correlator and standing wave generator device are used to convert frequency to phase information [6]. After that, the phase information is extracted and codded by phase comparison approach[7]. Single correlator schematic diagram is shown in Fig 1. The delay line in the graph sometimes can be phase shifter instead [8].

The microwave signal is divided into two roads in the same power. One of the two roads of signal went through the delayer, and the other didn't. After that, the correlator completed phase comparison, in fact, this also completed the transformation of the frequency information to the phase information. In theory, the delay line correlator implementation of instantaneous frequency measurement can reach the 
measuring accuracy better than $1 \mathrm{MHz}$. The measurement accuracy depends on the performance of each circuit constituting the system. For a fixed frequency, the longer the length of the delay line was, the higher the precision of frequency measurement can get.

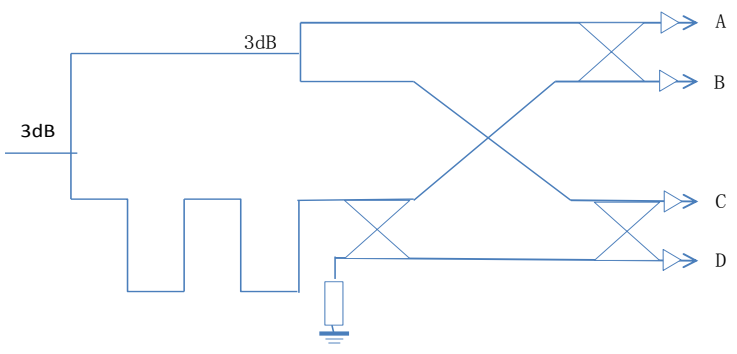

Figure.1 Single correlator schematic diagram

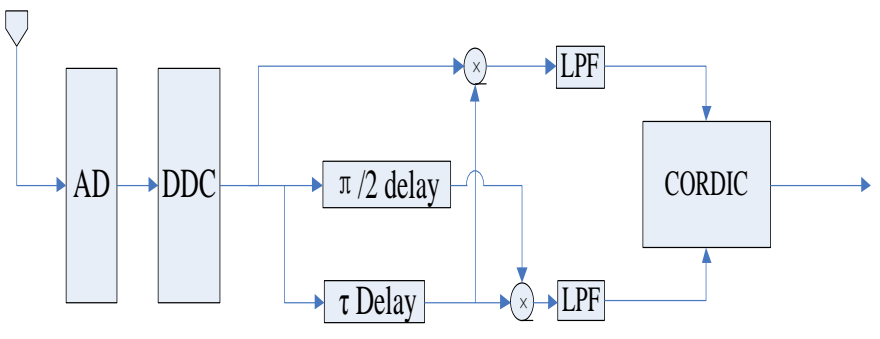

Figure. 2 Digital implementation of the digital frequency measurement schematic diagram

Digital Implementation.As shown in Fig 2, the steps of the digital method to implement the delay related frequency measurement are as follows:

(1) Radio frequency signal is transformed into digital signal by sampling and frequency conversion;

(2) Three way with the same frequency signals are got after the 90 degree phase shift and time delay $\tau$ respectively;

(3) The phase difference of the cosine component is gotten after the multiplication of the first and the third way signal passing through the low pass filter. By the same token, the phase difference of the sinusoidal component can be gotten by the second and the third way signal;

(4) The tangent value of the phase difference can be gotten after two orthogonal signals implementing the CORDIC algorithm. On this basis, we can get the value of the phase difference.

We assumed that the digital signal after the digital down converter (DDC) is:

$$
s(n)=A \sin \left(\omega n T_{s}\right)
$$

After $\frac{\pi}{2}$ phase shift: $\quad s_{1}(n)=A \sin \left(\omega n T_{s}+\frac{\pi}{2}\right)=A \cos \left(\omega n T_{s}\right)$

After $\tau$ phase shift: $\quad s_{2}(n)=A \sin \left(\omega n T_{s}-\omega \tau\right)$

We assumed that the phase difference: $\Delta \varphi=\omega \tau=2 \pi f \tau$

Then we do multiplication:

$$
\begin{aligned}
& s_{E}=s(n) s_{2}(n)=A^{2} \sin \left(\omega n T_{s}\right) \cdot \sin \left(\omega n T_{s}-\omega \tau\right)=\frac{A^{2}}{2}\left[\sin \left(2 \omega n T_{s}-\Delta \varphi\right)-\cos \Delta \varphi\right] \\
& s_{C}=s_{1}(n) s_{2}(n)=A^{2} \cos \left(\omega n T_{s}\right) \cdot \sin \left(\omega n T_{s}-\omega \tau\right)=\frac{A^{2}}{2}\left[\sin \left(2 \omega n T_{s}-\Delta \varphi\right)-\sin \Delta \varphi\right]
\end{aligned}
$$

After the low pass filter and filtering out the double frequency component, we got two orthogonal signals:

$$
s_{I}=\cos \Delta \varphi ; s_{Q}=\sin \Delta \varphi
$$

Next, we make CORDIC arithmetic on the two orthogonal signals. The basic formula of CORDIC algorithm is as follows. In the formulas, $\left(x_{i}, y_{i}\right)$ is the input vector, $\left(x_{i+1}, y_{i+1}\right)$ is the output vector, $\varphi_{i}$ is 
the rotation angle, $d_{i}$ is the direction of rotation of each iteration. Rotary's goal is to make $y$ converge to zero. $n \rightarrow 0,\left|y_{n} \rightarrow 0\right|, \quad z_{\infty} \rightarrow \Delta \varphi=\tan ^{-1}\left(y_{1} / x_{1}\right)$, the input vector phase extraction was completed. Here, we make $\left(s_{I}, s_{Q}\right)$ the input vector.

$$
\left\{\begin{array}{c}
x_{i+1}=x_{i}+d_{i} \cdot 2^{-i} \cdot y_{i} \\
y_{i+1}=y_{i}+d_{i} \cdot 2^{-i} \cdot x_{i} \\
z_{i+1}=z_{i}-d_{i} \cdot \alpha_{i} \\
\varphi=-\sum_{i=1}^{n} d_{i} \alpha_{i}, \varphi_{i}=\tan ^{-i}\left(2^{-i}\right), d_{i}=\operatorname{sign}\left(z_{i}\right), d_{i} \in\{-1,1\}
\end{array}\right.
$$

According to Eq.4, we can get $f: f=\frac{\Delta \varphi}{2 \pi \tau}$

It is important to note that $\left(s_{I}, s_{Q}\right)$ is the sine and cosine function of $\Delta \varphi$ respectively. Therefore, when $\Delta \varphi$ makes cycle changes, the phase output also can produce periodic changes [9]. According to the plus or minus sign of the sine and cosine of $\Delta \varphi$, we can calculate it accurately.

$$
\left\{\begin{array}{l}
\mathrm{I} \geq 0, \mathrm{Q} \geq 0, \Delta \varphi=\tan ^{-1}(I / Q) \\
\mathrm{I} \leq 0, \mathrm{Q} \geq 0, \Delta \varphi=\tan ^{-1}(I / Q)+\pi \\
\mathrm{I} \leq 0, \mathrm{Q} \leq 0, \Delta \varphi=\tan ^{-1}(I / Q)+\pi \\
\mathrm{I} \geq 0, \mathrm{Q} \leq 0, \Delta \varphi=\tan ^{-1}(I / Q)+2 \pi
\end{array}\right.
$$

\section{Experimental Results}

In the testing, we use the single frequency sine signal with white gaussian noise to simulate the method. As shown in Fig 3, we use MATLAB to generate the data points with the frequency of $30 \mathrm{MHz}$. Due to the phase calculation method for frequency measurement is very sensitive to the effects of the noise, so the precision frequency can be obtained by multi-point average [10].

Figure 4 shows the analysis of the effect of $\tau$ on the frequency measurement results. At this point, the signal-to-noise ratio (SNR) is $5 \mathrm{~dB}$, the data points is 1024 . Which can be seen from the diagram is that as the $\tau$ changes, the mean square value of the frequency measurement error also changes periodically. The closer the $\tau$ gets to the integer times of $\pi$, the smaller the mean square value error is. 

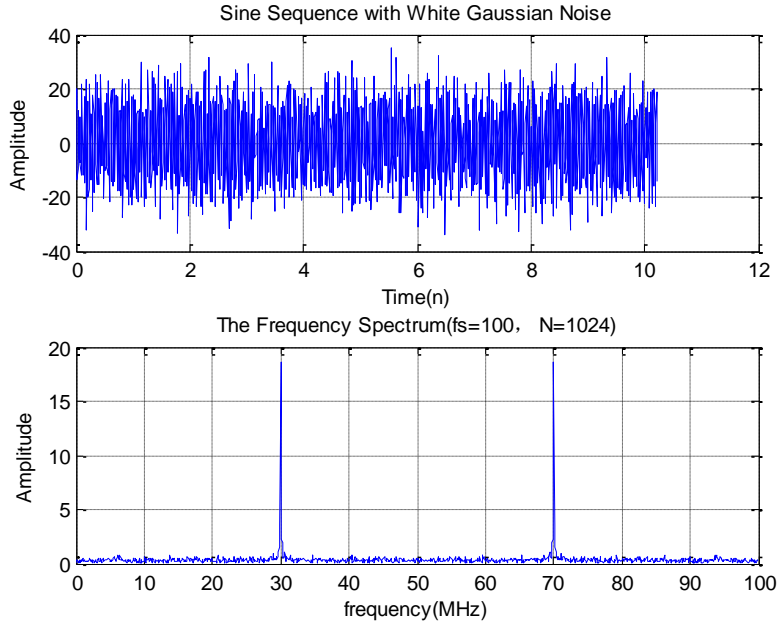

Fig.3 Single frequency signal for testing

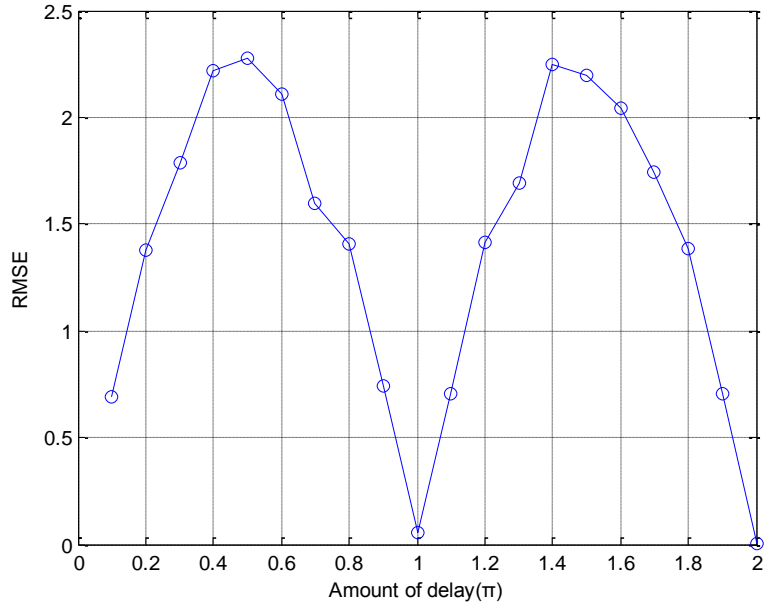

Fig.4 The relationship between the frequency measurement error and the amount of delay

Figure 5 shows the analysis of the effect of the SNR on the results. At this point, the amount of delay is $0.6 \pi$, the data points is 1024 . As we have seen, when the SNR reaches $5 \mathrm{~dB}$, the mean square error (MSE) of the results at about 0.5. With the further increase of the SNR, the RMSE value will further decrease. We made many times of simulations for different signals with different SNR and the amount of delay. Which can be seen from the data is that this method can get good phase and frequency estimation. Table 1 shows a set of the frequency measurement data of one of the experiment results. At this point, the data points are 1024 points. SNR is $5 \mathrm{~dB}$ and the amount of phase delay is $0.6 \pi$. As we can see from the data, the instantaneous frequency measurement precision is basically around $0.1 \mathrm{MHz}$. Pulse frequency measurement precision can reach within $100 \mathrm{~Hz}$ after a multipoint average operation.

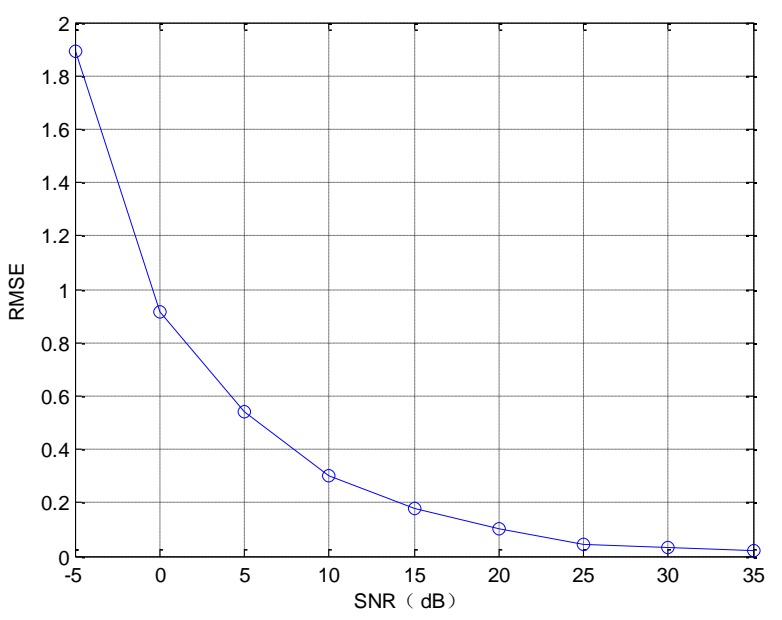

Fig.5. The effect of SNR for frequency measuring error 
Table 1 A single experimental result data

\begin{tabular}{|l|l|l|}
\hline $\begin{array}{l}\text { Experimental } \\
\text { parameters } \\
\text { SNR=5dB;N=1024 }\end{array}$ & $\begin{array}{l}\text { Frequency } \\
\text { measurement } \\
\text { (MHZ) }\end{array}$ & $\begin{array}{l}\text { Measurement } \\
\text { error (MHZ) }\end{array}$ \\
\hline 1 & 30.1823266429257 & 0.182326642925702 \\
\hline 2 & 29.7686337204989 & -0.231366279501099 \\
\hline 3 & 30.1253516449467 & 0.125351644946700 \\
\hline 4 & 29.8212577279969 & -0.178742272003102 \\
\hline$\ldots$ & $\ldots$ & $\ldots$ \\
\hline 1022 & 29.9689710455263 & -0.031028954473701 \\
\hline 1023 & 29.9310906009427 & -0.0689093990573007 \\
\hline 1024 & 30.0097048548677 & 0.009704854867701 \\
\hline RMSE & 0.037950644425216 & \\
\hline
\end{tabular}

\section{Discussion and Conclusion}

This paper puts forward a digital implementation of delay frequency measurement based on the CORDIC algorithm. This method avoids the use of multiplier, which can reduce multiplication resources in the FPGA implementations. This method can be helpful for engineering implementation. Due to the existence of nonlinear computation, this algorithm is only applicable to the frequency measurement for the high SNR signals. The simulation results show that under the condition of the SNR is greater than $5 \mathrm{~dB}$, this method has better performance.

\section{References}

[1] R. Williams, "Theroy and Application of a 0.5 to $18 \mathrm{GHz}$ Tunable IFM Receiver," Microwave Journal. USA, pp. 89-108, Feb 1989.

[2] J. Tsui, Microwave Receivers with Electronic Warfare Applications. New York: Wiley, 1986.

[3] Piotr. D. A High-Resolution CMOS Time to digital Converter Utilizing a Vernier Delay Line. IEEE Journal of Solid-State Circuits, 2000,35(2):240-247.

[4] P.W., "Design techniques and performance of digital IFM," Communications, Radar and Signal Processing, IEE Proceedings F, vol.129, no.3, pp.154-163, June 1982.

[5] Messer, H., "Wideband Instantaneous Frequency Measurements (IFM) Using SAW Devices," 1980 Ultrasonics Symposium, pp.226-229, 1980.

[6] Zhou W, Wang H. Development of the measurement and control technique in the time and frequency. Journal Of Time And Frequency.2003,26(2),87-95.

[7] L. Hu, Instantaneous Frequency Measurement, vol. 4. Bei Jing: National Defence Industry Press, 2002, pp.77-100. (In Chinese)

[8] G. Tian. "Time-Frequency Measurement for Sine Sweep Vibration Tests," 2005. (In Chinese)

[9] J. Yu. A Novel Super-High Resolution Phase Comparison Approach. PHYS. LETT. Vol.29,No. 7 .

[10] M. Tur and B. Moslehi, "Theory of laser phase noise in recirculating fiber-optic delay lines," J. Lightw. Technol., vol. LT-3, no. 1, pp. 20-31,Feb. 1985. 